\title{
Dynamic Output Feedback Consensus Control of Multi-agent Systems with Time-delay
}

\author{
Man Sun ${ }^{a}$ and Jing Gao \\ Department of Control Engineering, Northeastern University at Qinhuangdao, Qinhuangdao 066000, \\ Hebei, China \\ asun7661@126.com, brixinyueyi2014@126.com
}

Keywords: multi-agent, output-feedback, consensus control, time delay

\begin{abstract}
This paper considers the consensus problem of a multi-agent system with time delay and proposes a distributed dynamic output feedback protocol. A sufficient condition in terms of linear matrix inequalities (LMIs) is given to ensure consensus of the multi-agent system.
\end{abstract}

\section{Introduction}

Over the past few decades, the consensus problem in multi-agent systems has received considerable attention from many fields such as physics, biology and control theory. And numerous results have been obtained for consensus problems of multi-agent systems. For example, a simple model is proposed in [1] for phase transition of a group of self-driven particles and complex dynamics of the model are demonstrated numerically. Jadbabaie et al. ${ }^{[2]}$ studied the observed behavior reported in [1] and analyzed the alignment of an undirected network of agents with switching topologies which are periodically connected. Moreau[3] researched the nonlinear discrete-time multi-agent systems with time-dependent communication links, and introduced a novel method based on the notion of convexity. Olfati et al. [4] investigated a systematical framework of consensus problems with directed networks and undirected networks with fixed and switching topologies and time-delay and fixed topology, respectively. Later, Ren et al. [5] extended the conclusions of [2] and [4] and presented more loose conditions for consensus of information under dynamically changing interaction topologies.Owing to constraints on measurement or economic costs in practice, it is sometimes hard to measure the relative information of all states directly. However, only the relative information of all outputs is available. Thus, consensus protocols with dynamic output feedback (dynamic compensator) case are adopted in this paper. Because of each agent has limited capability of collecting information, the consensus protocol for each agent in descriptor multi-agent systems is distributed and only depends on the output information of the agent itself and its neighbours. Some researchers have investigated the consensus problem of the multi-agent systems through dynamic output-feedback approach ${ }^{[6]-[7]}$. However, the results about the consensus problem of the multi-agent systems with time delay through dynamic output-feedback approach are much less. In fact, time delays are ubiquitous phenomenon in multi-agent systems due to the congestion of communication channels and the finite transmission speed, which makes the dynamical behaviors of a network much more complicated. So it is necessary to consider the consensus problem of uncertain multi-agent systems with nonlinear dynamics and time delays.

\section{Preliminaries}

Let $\ell(v, \varepsilon, \rho)$ be an undirected graph of order $n$ with the set of nodes $v=\left\{s_{1}, \cdots, s_{n}\right\}$, the set of undirected edges $\varepsilon \subseteq v \times v$, and a symmetric adjacency matrix $\rho=\left\lfloor a_{i j}\right\rfloor$, with nonnegative elements $a_{i j}$. The node indexes belong to a finite index set $\{1, \cdots, n\}$. The adjacency elements associated with edges of graph are positive, that is, $e_{i j} \in \varepsilon$ or $e_{j i} \in \varepsilon$ if and only if $a_{i j}=a_{j i}>0$. Moreover, it is 
assumed that $a_{i i}=0$ for all $i \in\{1, \cdots, n\}$. The Laplacian corresponding to the graph $\ell$ is defined as $L=\left\lfloor l_{i j}\right\rfloor$, where $l_{i i}=\sum_{j} a_{i j}$, and $l_{i j}=-a_{i j}, i \neq j$.

Lemma $1^{[8]}$ : If an undirected graph $\ell$ is connected, then its Laplacian $L$ has the following properties:

1. Zero is a simple eigenvalue of $L$, and $1_{n}$ is the corresponding eigenvector, that is, $L 1_{n}=0$.

2. The rest $n-1$ eigenvalues are all positive.

Lemma $2^{[9]}$ : Let $L_{c}=\left\lfloor L_{c_{i j}}\right\rfloor \in R^{n \times n}$ be a symmetric matrix with $L_{c_{i j}}= \begin{cases}(n-1) / n, & i=j \\ -1 / n, & i \neq j\end{cases}$

Then the following statements hold:

1. The eigenvalues of $L_{c}$ are 1 with multiplicity $n-1$ and 0 with multiplicity 1 . The vectors $1_{n}^{T}$ and $1_{n}$ are the left and the right eigenvectors of $L_{c}$ associated with the zero eigenvalue, respectively.

2. There exists an orthogonal matrix $U=\left[\begin{array}{ll}U_{1} & \bar{U}_{1}\end{array}\right] \in R^{n \times n}$ with $\bar{U}_{1}=\frac{1}{\sqrt{n}} 1_{n}$, such that $U^{T} L_{c} U=\left[\begin{array}{cc}I_{n-1} & 0 \\ 0 & 0\end{array}\right]$ holds. Let $L \in R^{n \times n}$ be the Laplacian matrix of any given undirected graph, then $U^{T} L U=\left[\begin{array}{cc}L_{1} & 0 \\ 0 & 0\end{array}\right]$ holds, where $L_{1} \in R^{(n-1) \times(n-1)}$ is positive definite if and only if the corresponding graph is connected.

Consider the multi-agent system consisting of $n$ identical agents. Suppose the $i$ th agent has the following dynamics,

$$
\begin{aligned}
& \dot{x}_{i}(t)=A x_{i}(t)+B u_{i}(t) \\
& y_{i}(t)=C x_{i}(t), \quad i=1, \cdots, n
\end{aligned}
$$

where $x_{i}(t) \in R^{m}, u_{i}(t) \in R^{p}$ and $y_{i}(t) \in R^{q}$ are the state, the protocol and the measured output of the agent $i$, respectively.

Define $z_{i}(t)=x_{i}(t)-\frac{1}{n} \sum_{j=1}^{n} x_{j}(t), \quad i=1, \cdots, n$ as controlled output fuction to measure the disagreement of $x_{i}(t)$ to the average state of all agents. It is obvious that consensus of the multi-agent system can be achived if and only if $\lim _{t \rightarrow+\infty} z_{i}(t)=0$, for all $i=1, \cdots, n$.

Owing to constraints on measurement, it is sometimes hard to measure the relative information of all states directly. However, only the relative information of all outputs is available. Thus, consensus protocols with dynamic output feedback case are adopted in this paper.

We can design a following distributed dynamic output feedback protocol with time delay.

$$
\begin{aligned}
& \dot{v}_{i}(t)=A_{K} v_{i}(t)+B_{K} \sum_{j=1}^{n} a_{i j}\left(y_{i}(t-\tau)-y_{j}(t-\tau)\right) \\
& u_{i}(t)=C_{K} v_{i}(t)+D_{K} \sum_{j=1}^{n} a_{i j}\left(y_{j}(t-\tau)-y_{j}(t-\tau)\right), \quad i=1, \cdots, n
\end{aligned}
$$

where $v_{i}(t) \in R^{s}$ is the state of the dynamic output feedback controller, and $a_{i j}$ is the adjacency weights of the interaction graph $\ell$.

Substituting protocol (2) into the system (1), we can obtain the following closed loop system

$$
\begin{aligned}
& \bar{\xi}(t)=\bar{A} \bar{\xi}(t)+\bar{A}_{1} \bar{\xi}(t-\tau) \\
& z(t)=\bar{C} \bar{\xi}(t) \\
& \text { where } \bar{A}=\left[\begin{array}{cc}
I_{n} \otimes A & I_{n} \otimes B_{1} C_{K} \\
0 & I_{n} \otimes A_{K}
\end{array}\right], \bar{A}_{1}=\left[\begin{array}{cc}
L \otimes B_{1} D_{K} C & 0 \\
L \otimes B_{K} C & 0
\end{array}\right], \bar{C}=\left[\begin{array}{ll}
L_{c} \otimes I_{m} & 0
\end{array}\right]
\end{aligned}
$$


$v(t)=\left[v_{1}^{T}(t) \cdots v_{n}^{T}(t)\right]^{T}, \quad \bar{\xi}(t)=\left[\begin{array}{ll}x^{T}(t) & v^{T}(t)\end{array}\right]^{T}, L$ is the Laplacian of graph $\ell$.

We can see that $L$ is a singular matrix, therefore the closed loop system is uncontrollable if the given matrix $A$ is unstable. So we must make a model transformation of the closed loop system.

Let $\hat{x}(t)=x(t)-1_{n} \otimes \frac{1}{n} \sum_{j=1}^{n} x_{j}(t)=\left(L_{c} \otimes I_{m}\right) x(t), \hat{v}(t)=\left(L_{c} \otimes I_{s}\right) v(t), \hat{x}(t-\tau)=\left(L_{c} \otimes I_{m}\right) x(t-\tau)$.

Notice $L_{c} 1_{n}=0, L 1_{n}=0$. Then the closed loop system (3) can be rewrite the following form.

$$
\begin{aligned}
& \hat{\xi}(t)=\hat{A} \hat{\xi}(t)+\hat{A}_{1} \hat{\xi}(t-\tau) \\
& z(t)=\hat{C} \hat{\xi}(t)
\end{aligned}
$$

where $\hat{A}=\left[\begin{array}{cc}L_{c} \otimes A & L_{c} \otimes B_{1} C_{K} \\ 0 & L_{c} \otimes A_{K}\end{array}\right], \hat{A}_{1}=\left[\begin{array}{cc}L_{c} L \otimes B_{1} D_{K} C & 0 \\ L_{c} L \otimes B_{K} C & 0\end{array}\right], \hat{C}=\left[\begin{array}{ll}L_{c} \otimes I_{m} & 0\end{array}\right]$

$U^{T} L_{c} U=\left[\begin{array}{cc}I_{n-1} & 0 \\ 0 & 0\end{array}\right], \quad U^{T} L U=\left[\begin{array}{cc}L_{1} & 0 \\ 0 & 0\end{array}\right]$

Let $\tilde{x}(t)=\left(U^{T} \otimes I_{m}\right) \hat{x}(t), \tilde{v}(t)=\left(U^{T} \otimes I_{s}\right) \hat{v}(t), \tilde{z}(t)=\left(U^{T} \otimes I_{m}\right) z(t)$.

Then the closed loop system (4) can be rewrite the following form.

$$
\begin{aligned}
& \tilde{\xi}(t)=\tilde{A} \tilde{\xi}(t)+\tilde{A}_{1} \tilde{\xi}(t-\tau) \\
& \tilde{z}(t)=\tilde{C} \tilde{\xi}(t)
\end{aligned}
$$

where $\quad \tilde{A}=\left[\begin{array}{cc}U^{T} L_{c} U \otimes A & U^{T} L_{c} U \otimes B_{1} C_{K} \\ 0 & U^{T} L_{c} U \otimes A_{K}\end{array}\right] \quad, \quad \tilde{A}_{1}=\left[\begin{array}{cc}U^{T} L_{c} L U \otimes B_{1} D_{K} C & 0 \\ U^{T} L_{c} L U \otimes B_{K} C & 0\end{array}\right]$, $\widetilde{C}=\left[\begin{array}{ll}U^{T} L_{c} U \otimes I_{m} & 0\end{array}\right]$

\section{Main results}

Then we can derive an reduced-order system as follows.

$$
\begin{aligned}
& \dot{\xi}^{\prime}(t)=A^{\prime} \xi^{\prime}(t)+A_{1}^{\prime} \xi^{\prime}(t-\tau) \\
& z^{\prime}(t)=C^{\prime} \xi^{\prime}(t)
\end{aligned}
$$

where, $A^{\prime}=\left[\begin{array}{cc}I_{n-1} \otimes A & I_{n-1} \otimes B_{1} C_{K} \\ 0 & I_{n-1} \otimes A_{K}\end{array}\right], A_{1}^{\prime}=\left[\begin{array}{cc}L_{1} \otimes B_{1} D_{K} C & 0 \\ L_{1} \otimes B_{K} C & 0\end{array}\right], C^{\prime}=\left[\begin{array}{ll}I_{n-1} \otimes I_{m} & 0\end{array}\right]$

Theorem 1: Consider the network with an undirected interaction graph $\ell$ that is connected. The system (6) is asymptotically stable, if there exist a dynamic output feedback $u(t)$ and positive definite matrices $P_{i} \in R^{(m+s) \times(m+s)}, i=1, \cdots, n-1$, such that the following LMIs are satisfied for $i=1, \cdots, n-1$.

$$
\left.\Xi=\left[\begin{array}{cc}
A & B_{1} C_{K} \\
0 & A_{K}
\end{array}\right]_{*}^{T} P+P\left[\begin{array}{cc}
A & B_{1} C_{K} \\
0 & A_{K}
\end{array}\right]+S \quad P\left[\begin{array}{cc}
\lambda_{i} B_{1} D_{K} C & 0 \\
\lambda_{i} B_{K} C & 0 \\
-S
\end{array}\right]\right]<0
$$

Proof: By Lemma 2, the matrix $L_{1}$ is positive definite. Therefore, there exists an orthogonal matrix $Q \in R^{(n-1) \times(n-1)} \quad$ such that $Q^{T} L_{1} Q=\operatorname{diag}\left\{\lambda_{1}, \cdots, \lambda_{n-1}\right\}$. Let $x^{\prime \prime}(t)=\left(Q^{T} \otimes I_{m}\right) x^{\prime}(t)$, $v^{\prime \prime}(t)=\left(Q^{T} \otimes I_{s}\right) v^{\prime}(t), z^{\prime \prime}(t)=\left(Q^{T} \otimes I_{m}\right) z^{\prime}(t)$. Then the system (6) can be rewrited in the following form.

$$
\begin{aligned}
& \dot{\xi}^{\prime \prime}(t)=A^{\prime \prime} \xi^{\prime \prime}(t)+A_{1}^{\prime \prime} \xi^{\prime \prime}(t-\tau) \\
& z^{\prime \prime}(t)=C^{\prime \prime} \xi^{\prime \prime}(t)
\end{aligned}
$$


where, $A^{\prime \prime}=\left[\begin{array}{cc}I_{n-1} \otimes A & I_{n-1} \otimes B_{1} C_{K} \\ 0 & I_{n-1} \otimes A_{K}\end{array}\right], A_{1}^{\prime \prime}=\left[\begin{array}{cc}\operatorname{diag}\left\{\lambda_{1}, \cdots \lambda_{n}\right\} \otimes B_{1} D_{K} C & 0 \\ \operatorname{diag}\left\{\lambda_{1}, \cdots, \lambda_{n}\right\} \otimes B_{K} C & 0\end{array}\right], C^{\prime \prime}=\left[\begin{array}{ll}I_{n-1} \otimes I_{m} & 0\end{array}\right]$.

We can decompose the system (7) to the following $n-1$ subsystems.

$$
\begin{aligned}
& \dot{\xi}_{i}^{\prime \prime}(t)=A_{i}^{\prime \prime} \xi_{i}^{\prime \prime}(t)+A_{1 i}^{\prime \prime} \xi_{i}^{\prime \prime}(t-\tau), i=1, \cdots, n \\
& z_{i}^{\prime \prime}(t)=C_{i}^{\prime \prime} \xi_{i}^{\prime \prime}(t)
\end{aligned}
$$

where, $A_{i}^{\prime \prime}=\left[\begin{array}{cc}A & B_{1} C_{K} \\ 0 & A_{K}\end{array}\right], A_{1 i}^{\prime \prime}=\left[\begin{array}{cc}\lambda_{i} \otimes B_{1} D_{K} C & 0 \\ \lambda_{i} \otimes B_{K} C & 0\end{array}\right], C_{i}^{\prime \prime}=\left[\begin{array}{ll}I_{m} & 0\end{array}\right]$

If for all $i=1, \cdots, n$, system (8) is asymptotically stable, system (6) is asymptotically stable.

Consider the following Lyapunov-Kasovskii function,

$V\left(\xi_{i}^{\prime \prime}(t)\right)=\xi_{i}^{\prime \prime}(t) P \xi_{i}^{\prime \prime}(t)+\int_{t-\tau}^{t} \xi_{i}^{\prime T}(s) S \xi_{i}^{\prime \prime}(s) d s$

If $\Xi<0$, we can obtain that $\dot{V}\left(\xi_{i}^{\prime \prime}(t)\right)<0$.

\section{Acknowledgements}

This work has been supported by the National Natural Science Foundation of China under Grant No.61403069, Natural Science Foundation of Hebei Province under Grant No. F2014501055 and F2012501044, the Program of Science and Technology Research of Hebei University No. ZD20132003, the Program for New Century Excellent Talents in University No. NCET-12-0103.

\section{Summary}

The consensus problem of a multi-agent system with time delay is dealt with through a distributed dynamic output feedback protocol. A sufficient condition in terms of linear matrix inequalities (LMIs) is obtained to ensure consensus of the multi-agent system.

\section{References}

[1] T. Vicsek, A. Cziro' ok, E. Ben-Jacob, etc., "Novel type of phase transition in a system of self-deriven particles”, Physical Review Letters, Vol. 75 (1995), no. 6, p. 1226-1229.

[2] A. Jadbabaie, J. Lin, and A. S. Morse, "Coordination of groups of mobile autonomous agents using nearest neighbor rules”, IEEE Trans. on Auto. Control, Vol. 48 (2003), no. 6, p. 988-1001.

[3] L. Moreau, "Stability of multi-agent systems with time-dependent communication links", IEEE Trans. on Automatic Control, Vol. 50 (2005), no.2, p. 169-182.

[4] R. Olfati-Saber, and R. M. Murray, “Consensus problems in networks of agents with switching topology and time-delays”, IEEE Trans. On Auto. Control, Vol. 49 (2004), no. 9, p. 1520-1533.

[5] W. Ren and R. W. Beard, "Consensus seeking in multi-agent systems under dynamically changing interaction topologies”, IEEE Trans. On Automatic Control, Vol. 50 (2005), no. 5, p. 655-661.

[6] X. Yang and G. Liu, “Consensus of descriptor multi-agent systems via dynamic compensators”, IET Control Theory and Applications, Vol. 8 (2014), no. 6, p. 389-398.

[7] Y. Liu, Y. Jia, J. Du and S. Yuan, "Dynamic Output Feedback Control for Consensus of Multi-agent Systems: An $H_{\infty}$ Approach”, American Control Conference, p. 4470-4475, (2009).

[8] R. Olfati-Saber and R. M. Murray, "Consensus problems in networks of agents with switching topology and time-delays”, IEEE Trans. on Auto. Control, Vol. 49 (2004), no. 9, p.1520-1533.

[9] P. Lin and Y. Jia, "Average consensus in networks of multi-agents with both switching topology and coupling time-delay”, Physica A, Vol. 387 (2008), no. 1, p. 303-313. 